\title{
Polymer Surface Diffusion in the Dilute Limit
}

\author{
Janet S. S. Wong \\ Department of Mechanical Engineering, Imperial College, London U.K.
}

\author{
Liang Hong, ${ }^{a}$ Sung Chul Bae, ${ }^{a}$ and Steve Granick ${ }^{\mathrm{a}, \mathrm{b}}$ \\ Departments of Materials Science and Engineering, ${ }^{\mathrm{a}}$ Chemistry, ${ }^{\mathrm{b}}$ and Physics ${ }^{\mathrm{b}}$ \\ University of Illinois, Urbana, IL 61801
}

\begin{abstract}
$\underline{\text { Abstract }}$
The molecular weight (M) dependence of the surface diffusion coefficient (D) is measured for polystyrene adsorbed from chloroform, a good solvent, in the dilute limit of surface coverage. On as-received smooth quartz surfaces, we reproduce $D \sim M^{-3 / 2}$, the same power law observed earlier in aqueous systems for a much smaller range of M. This is consistent with computer simulations in the literature regarding $2 \mathrm{D}$ diffusion between obstacles. But on smoother surfaces, mica and thermally annealed quartz, we observe Rouse scaling, $D \sim M^{-1}$, to our knowledge the first time this theoretically-expected scaling has been observed for polymer diffusion at the solid-liquid interface. For polystyrene of the highest molecular weight diffusing on the rougher surfaces, in the range 1 to $6.7 \times 10^{5} \mathrm{~g}-\mathrm{mol}^{-1}$, the dependence on $\mathrm{M}$ softens to weaker than a power law of $-3 / 2$, suggesting reversion to Rouse behavior.
\end{abstract}


$\underline{\text { Introduction }}$

It may seem paradoxical that polymer physics has not yet resolved the problem of surface diffusion; after all, diffusion has long occupied a large part of the agenda in polymer physics as concerns bulk diffusion [1,2]. The difficulty is to quantify it and at surfaces, in the dilute limit that is simplest conceptually, this requires single-molecule measurements. Here we are interested in scaling behavior: how the surface translational diffusion coefficient $(D)$ scales with molecular weight $(M)$ of a flexible chain. We pose this question because as the adsorption energy of polymers is typically so slight per segment $\left(\sim \mathrm{k}_{\mathrm{B}} \mathrm{T}\right)$ yet enormous in a chain molecule comprised of numerous segments, the essence of the problem is to understand how chain length matters.

To summarize limitations of the present state of knowledge: First, for diffusion on a homogeneous solid surface, theoretical considerations predict $D \sim M^{-1}$ ("Rouse diffusion") because friction scales linearly with molecular weight $[3,4]$ (the limits have been explored in simulations [5-7]); but the operational limits of homogeneity needed to achieve this in an experiment are not known, and even a single crystal surface possesses not only structure but also defects. Secondly, it is known that surface disorder can in principle guide chains to diffuse between obstacles [8,9], the resulting reptation-type diffusion scaling as $D \sim M^{-3 / 2}$ [10-12] but the limits to which this holds for chains that may not be strictly two-dimensional owing to their weak segmental sticking energy are not known. Third, when chains possess extremely high $M$ such that 2D diffusion becomes extremely slow, it is evident that other mechanisms might become rate-limiting; but this possibility has not been considered previously, to the best of our knowledge. 
This study addresses all of these questions. It goes beyond the limitations of a previous study from this laboratory $[13,14]$ where a narrow range of $M$ was considered and questions of system-specificity were presented by the choice of an aqueous system. Here we consider scaling with $M$ over an exceptionally large span of molecular weight while investigating polystyrene, whose nonpolar character renders it a model system in which to investigate generic effects. We avoid the complexity of chain-chain interactions that would occur outside the dilute limit [1519].

\section{Experimental Section}

To obtain single-molecule sensitivity, we employ fluorescence correlation spectroscopy (FCS) [20]. While there would be no dearth of fluorescent dyes suited for experiments in aqueous solution, to study polystyrene in nonpolar solvent was trickier experimentally. We screened numerous dye-solvent combinations before selecting the uncharged dye, bodipy-FL (Molecular Probes); it is soluble in chloroform, which in turn is a convenient good solvent for polystyrene (PS), yet this dye does not adsorb from chloroform to the surfaces that we investigated. It was linked chemically to one sole end of amino-terminated or carboxylterminated PS (Polymer Source, Inc.) whose characteristics are summarized in Table I. The FCS measurements were made in a home-built apparatus using a Zeiss $63 \mathrm{x}$ air objective with N.A. $=0.75$, laser excitation at $488 \mathrm{~nm}$, and a diffraction-limited excitation spot $(\approx 350 \mathrm{~nm}$ diameter). The possibility that chains diffuse by hopping off the surface, then re-adsorb, is negated by the finding that $D$ depends on $M$ as a power law, weaker than the exponential dependence anticipated for a process rate-limited by desorption [21]. For each polymer sample, measurements were made at 2-3 dozen different spots on the surface. The histograms in Fig. 1, for two such families of experiments, illustrate excellent repeatability as $D$ varies by 3 orders of 
magnitude. These data are consistent with a single diffusion process, as may be seen in the autocorrelation curve in Fig. 1, where the line through the data is the expectation for Fickian diffusion with the surface diffusion coefficient as the sole fitting parameter.

To prepare experiments, chains were first allowed to adsorb from dilute (nM) chloroform solution for $\sim 1 \mathrm{hr}$, then the solution was rinsed repeatedly with pure solvent and finally the experiment was allowed to equilibrate for $8 \mathrm{hr}$ before starting measurements. The surface coverage of $\sim 1$ molecule- $\mu \mathrm{m}^{-2}$ was confirmed from the short-time limit of FCS autocorrelation functions. The adsorbing solid was either muscovite mica, freshly cleaved, or quartz prepared as described below. The laser power at the sample was kept as low as $60 \mu \mathrm{W}$ to prevent heating and potential photodegradation. This we verified in control experiments with other laser power, and is shown by internal consistency of the data in that a constant baseline of photon counts was observed throughout the duration of the experiments. Temperature was room temperature, 23-25 C.

\section{$\underline{\text { Results and discussion }}$}

In Fig. 2, $D$ is plotted against $M$ on log-log scales not only for diffusion on freshlycleaved mica after adsorption from dilute chloroform solution, but also for diffusion of these same polymers in dilute bulk solution. The measurements in dilute bulk solution obey expected scaling [1,2], lending confidence in the experimental approach. The measurements of surface diffusion, showing that the magnitude of $D$ is at least 2 orders of magnitude smaller, may help to explain why the polymer literature abounds in arguments whether polymer adsorption equilibrates. We also confirmed this finding using a second solid surface, quartz that we annealed extensively in our laboratory at $1210^{\circ} \mathrm{C}$ to first smoothen it (Fig. 4) [22]. Simulations 
predict this $D \sim M^{-1}$ scaling for thermally-activated diffusion at equilibrium [3], but to the best of our knowledge it has not been previously reported in experiments on solids.

Although scaling with $M$ is the same, on mica and on annealed quartz, we also emphasize differences. On quartz, adsorption is so weak that polymers of the lowest $M\left(2,500 \mathrm{~g}-\mathrm{mol}^{-1}\right)$ desorb into pure solvent over the span of $\sim 1 \mathrm{hr}$ after chains in solution are washed away (this is why data for this $M$ is not presented; it precludes meaningful FCS measurements). A higher segmental adsorption energy probably explains why $D$ on mica was smaller by an order of magnitude, for samples of the same molecular weight. While the classic de Gennes 'pancake' surface conformation is expected to hold for sound theoretical reasons [2] in a time-averaged sense, which would render the activation energy for every adsorbed segment to desorb simultaneously to be prohibitively large [21], failure of short chains to remain adsorbed onto quartz, when exposed to pure solvent, suggests that chain conformations fluctuate transiently into more loopy states and desorb from those states.

Furthermore, this quartz system provides the pathway to test the model that stronger scaling, $D \sim M^{-3 / 2}$, holds if obstacles force chains to diffuse in curvilinear fashion, which is what computer simulations of two-dimensional polymer diffusion predict [5-9]. To this end, we compared polymer diffusion on ultrasmooth (thermally annealed) quartz with diffusion on quartz that is less smooth, being only mechanically-polished. Both surfaces are prepared before experimentation using a cleaning protocol expected to give similar surface chemical makeup [23]. The distribution of surface roughness, compared using atomic force microscopy (AFM) with an exceptionally sharp tip is plotted in Fig. 3. One notices that both surfaces were exceptionally smooth with root-mean-square roughness $<1 \mathrm{~nm}$, yet the mechanically-polished surface is slightly rougher and somewhat skewed in its distribution of surface roughness. 
Limitations of current characterization technology preclude at this time better evaluation of the nanometer-scale heterogeneity on these surfaces. In principle, heterogeneity can be chemical as well as topographical. AFM adhesion experiments in air (not shown) failed to detect adhesion heterogeneity. However, whether this comprises the correct measure of surface heterogeneity is not clear; this measure of surface roughness normal to the plane may simply present shallow slopes on which the adsorbed polymers sit, which would not be expected to modify their diffusion.

Another measure of roughness would be distribution of chemical heterogeneity from spot to spot in the plane of the surface. In this scenario, polymers would experience obstacles to surface diffusion if their footprint were smaller than the characteristic length of such roughness, but would experience a more homogeneous environment if their footprint exceeded it. Estimated $2 \mathrm{D}$ radii of gyration provide an estimate, in this scenario, of the length scale of surface roughness. To this end, we estimate $R_{2 D}=a M^{0.75}$, for which there is good theoretical support [1], and for simplicity suppose the parameter $a$ to be the same as for this same polymer in the bulk solution (while on physical grounds it would be not unreasonable to suppose adsorption to stiffen the polymer chain, how much this might happen is not known). This calculation gives, from lowest to higher $M$ for the $M$ summarized in Table $I: R_{2 D} \approx 4,14,30,80,160,290 \mathrm{~nm}$. Then, from observing the break in $\mathrm{M}$ dependence at $\mathrm{M}=120, \mathrm{~g}-\mathrm{mol}^{-1}$, one might estimate the lateral roughness as $\approx 80 \mathrm{~nm}$. To test this line of argument, it would be an enticing thought to vary the surface roughness systematically in order to look for corresponding shifts of the break of $\mathrm{M}$ dependence, but this seems impractical at present, as to control roughness on nm length scales does not seem to be technically possible. 
In Fig. 4, $D$ for the same polymer samples is compared for the two quartz surfaces, asreceived and thermally annealed. Over the range of $M$ investigated in - previous experiments on this question $[13,14]$, data for the rougher surface are consistent with the $D \sim M^{-3 / 2}$ predicted by simulations [8-12,15], while data for diffusion on the smoother surface obey $D \sim M^{-1}$ observed previously only for diffusion on a fluid surface ( DNA on supported phospholipid bilayers [24,25]). As we observe $D \sim M^{-1}$ on both a crystalline surface (mica) and on an aperiodic surface (quartz) shows that explanation should not be sought in surface-specific structure or lack of structure, though this is the premise of some theoretical approaches [15]. Extrapolating the trend in the two sets of data to the point that the curves cross at small $\mathrm{M}$, one notices that this crossover occurs at the same point at which the smoother surface failed to adsorb polymer.

We now discuss the observation that the $-3 / 2$ power-law scaling in this data, weakened when the measurements were pushed to chains 5 times larger (Fig. 4). Till present it has not been possible to do experiments with samples of even higher $M$, as the needed sample must bear a functional endgroup at which to attach a fluorescent dye, and we have not found a source of such samples. Beyond $\approx 1 \times 10^{5} \mathrm{~g}-\mathrm{mol}^{-1}$, we observe reversion from $-3 / 2$ to weaker $M$ dependence - to scaling consistent with Rouse scaling though, as the range of $M$ is just a factor of 5 , the precise scaling power cannot be determined with confidence. Note that the prefactor of $D \sim M^{-1}$ scaling remains an order of magnitude slower than on the smoother surface.

This may reflect a change in the rate-limiting process. Chains below a critical $M$ may be expected to diffuse between surface obstacles as models predict for a two-dimensional chain [69], but there should come a point where this process is so slow that hopping over these obstacles becomes the faster process instead (still slow but not quite so slow). This would be consistent with the fact that chains with $M<3,000 \mathrm{~g}-\mathrm{mol}^{-1}$ fail to adsorb at all, showing that while the time- 
averaged conformation is the predicted pancake [2], transient loops also exist. These arguments lead one to expect the rate-limiting process to switch from a mechanism of curvilinear diffusion (low $M$ ) to surface hopping of transient loops over topographical obstacles (high $M$ ). We are not aware of theory that yet models this scenario and do not insist on it, as the main point of this paper is to present experimental data.

\section{$\underline{\text { Conclusions }}$}

This study clarifies kinetic regimes of polymer surface diffusion. In observing either $D$ $M^{-1}$ (observed here experimentally for what is considered to be the first time, for diffusion on a solid surface) or $D \sim M^{-3 / 2}$, depending on surface roughness, we quantify the amount of roughness needed - it is remarkably little -- to produce curvilinear surface diffusion. Beyond this, by observing re-entry to weaker $M$ dependence when $M$ is very large, these experiments show what is considered to be the first demonstration of a new kinetic regime of surface diffusion, though the precise power law dependence in this regime (and whether it is a power law, or some other functional relation) is uncertain owing to the limited range of $\mathrm{M}$ over which the effect could be studied. The results presented here have fundamental implications in setting new criteria for predicting the time scales of surface diffusion in the dilute limit, which we chose to address here because it is the cleanest conceptual limit.

For discussions, we thank J. F. Douglas, P. Keblinski, S. Kumar, M. Rubinstein, and K. Schweizer. This study was supported by the taxpayers of the U.S. through the National Science Foundation (Polymers Program), DMR-0907018. 


\section{References}

1. Rubinstein, M.; Colby, R. H. Polymer Physics; Oxford University Press: New York, 2003.

2. de Gennes, P.-G. Scaling Concepts in Polymer Physics: Cornell University Press: Ithaca, 1979.

3. Carmesin, I.; Kremer, K. J. Phys. (Paris) 1990, 51, 915.

4. Milchev, A.; Binder, K. Macromolecules, 1996, 29, 343.

5. Mukherji, D.; Bartels, G.; Muser, M. H. Phys. Rev. Lett. 2008, 100, 068301.

6. Desai, T. G.; Keblinski, P.; Kumar, S. K. J. Chem. Phys. 2008, 128, 044903.

7. Hoda, N.; Kumar, S. Phys. Rev. E 2009, 79, 020801.

8. Azuma, R.; Takayama, H. J. Chem. Phys. 1999, 111, 8666.

9. Slater, G. W.; Wu, S. Y. Phys. Rev. Lett. 1995, 75, 164.

10. Desai, T. P.; Keblinski, P.; Kumar, S. K.; Granick, S. J. Chem. Phys. 2006, 124, 084904.

11. Desai, T. P.; Keblinski, P.; Kumar, S. K.; Granick, S. Phys. Rev. Lett. 2007, 98, 218301.

12. Qian, H. -J.; Chen, L. -J.; Lu, Z. -Y.; Li, Z. -S. Phys. Rev. Lett. 2007, 99, 068301.

13. Sukhishvili, S. A.; Chen, Y.; Müller, J. D.; Gratton, E.; Schweizer, K. S.; Granick, S. Nature 2000, 406, 146.

14. Sukhishvili, S. A.; Chen, Y.; Müller, J. D.; Gratton, E.; Schweizer, K. S.; Granick, S. Macromolecules 2002, 35, 1776.

15. Mukherji, D.; Müser, M. H. Phys. Rev. E 2006, 74, 010601 (R).

16. Mukherji, D.; Bartels, G.; Müser, M. H. Phys. Rev. Lett. 2008, 100, 068301.

17. Zhao, J.; Granick, S. J. Am. Chem. Soc. 2004, 126, 6242.

18. Klein Wolterink, J.; Barkema, G. T.; Cohen Stuart, M. A. Macromolecules 2005, 38, 2009.

19. Zhao, J.; Granick, S. Macromolecules 2007, 40, 1243. 
20. Lakowicz, J. R. Principles of Fluorescence Spectroscopy; Springer: N.Y. 2006.

21. Johnson, H. E.; Granick, S. Science 1992, 255, 966.

22. In a tube furnace, temperature was increased at $300^{\circ} \mathrm{C}-\mathrm{hr}^{-1}$ to $1210^{\circ} \mathrm{C}$, kept at $1210^{\circ} \mathrm{C}$ for 3 $\mathrm{hr}$, then heat was turned off and the quartz slides were allowed to cool inside the furnace until they reached $100{ }^{\circ} \mathrm{C}$. They were then removed from the furnace, flipped to avoid excessive sagging, and the heating cycle was repeated. The total time at $1210^{\circ} \mathrm{C}$ was 42 to $48 \mathrm{hr}$.

23. Fused silica quartz slides, purchased from SPI Supplies, SuperSmooth ${ }^{\mathrm{TM}}$, were immersed in a base bath (mixture of potassium hydroxide and 2-propanol) for one day, rinsed and dried, exposed to oxygen plasma treatment for $20 \mathrm{~min}$, rinsed and dried, then mounted in the sample cell. The cell was filled with chloroform for $1 \mathrm{hr}$ then rinsed with chloroform. Finally, polystyrene was allowed to adsorb from dilute solution for an hour and rinsed with chloroform solvent.

24. Maier, B.; Rädler, J. O. Phys. Rev. Lett. 1999, 82, 1911.

25. Maier, B.; Rädler, J. O. Macromolecules 2002, 35, 1776. 
Table 1. Characteristics of the polystyrene samples as reported by the manufacturer.

\begin{tabular}{lll}
$\begin{array}{ll}\mathbf{M}_{\mathbf{n}} \\
\mathrm{kg}-\mathrm{mol}^{-1}\end{array}$ & & $\mathbf{M}_{\mathrm{w}} / \mathbf{M}_{\mathbf{n}}$ \\
\hline 670 & & 1.06 \\
300 & & 1.18 \\
120 & & 1.04 \\
32 & & 1.04 \\
12 & & 1.02 \\
2.5 & & 1.10
\end{tabular}




\section{Figure Captions}

Fig. 1. Histograms of translational diffusion coefficients (D) measured for $M=12,000$ (dark grey) and 670,000 g-mol ${ }^{-1}$ (light grey), after adsorption onto freshly-cleaved mica and subsequent rinsing with pure chloroform solvent. The inset shows a representative intensityintensity fluorescence autocorrelation curve, $\mathrm{G}(\tau) / \mathrm{G}(0)$, where $\tau$ is the correlation time, plotted against $\log (\tau)$, for PS with $\mathrm{M}=120,000 \mathrm{~g}-\mathrm{mol}^{-1}$. The spread in the histogram is reasonable given the molecular weight distribution (for example, for $\mathrm{M}_{\mathrm{w}} / \mathrm{M}_{\mathrm{n}}=1.05$, the standard deviation of $M_{n}$ equals $\left.(0.05)^{1 / 2} M_{n}=0.22 M_{n}\right)$.

Fig. 2. Center-of-mass diffusion coefficient (D) for surface diffusion on mica (squares) and for PS in dilute chloroform solution (circles), plotted against number-average molecular weight $\left(\mathrm{M}_{\mathrm{n}}\right)$ on log-log scales. Lines through the data are drawn with slopes -1 and -0.6 , respectively. Each datum is the average of 20-30 measurements.

Fig. 3. Histograms of relative surface roughness, in units of relative frequency per unit area and normalized to relative frequency of unity, for the two quartz surfaces studied, thermally annealed (hatched) and mechanically polished (grey). These were measured using AFM in tapping mode in repeated $2 \mu \mathrm{m} \times 2 \mu \mathrm{m}$ scans. The distributions differ distinctively though quantitative numbers may be convoluted with size of the AFM tip, stated by the manufacturer to be $2 \mathrm{~nm}$. The rms (root mean square) roughness was 0.17 and $0.55 \mathrm{~nm}$, respectively.

Fig. 4. Center-of-mass diffusion coefficient (D) for surface diffusion on annealed quartz (squares) and mechanically-polished quartz (circles), plotted against number-average molecular 
weight $\left(\mathrm{M}_{\mathrm{n}}\right)$ on log-log scales. As guides to the eye, lines through the data are drawn with slopes -1 and $-3 / 2$. 
Figure 1

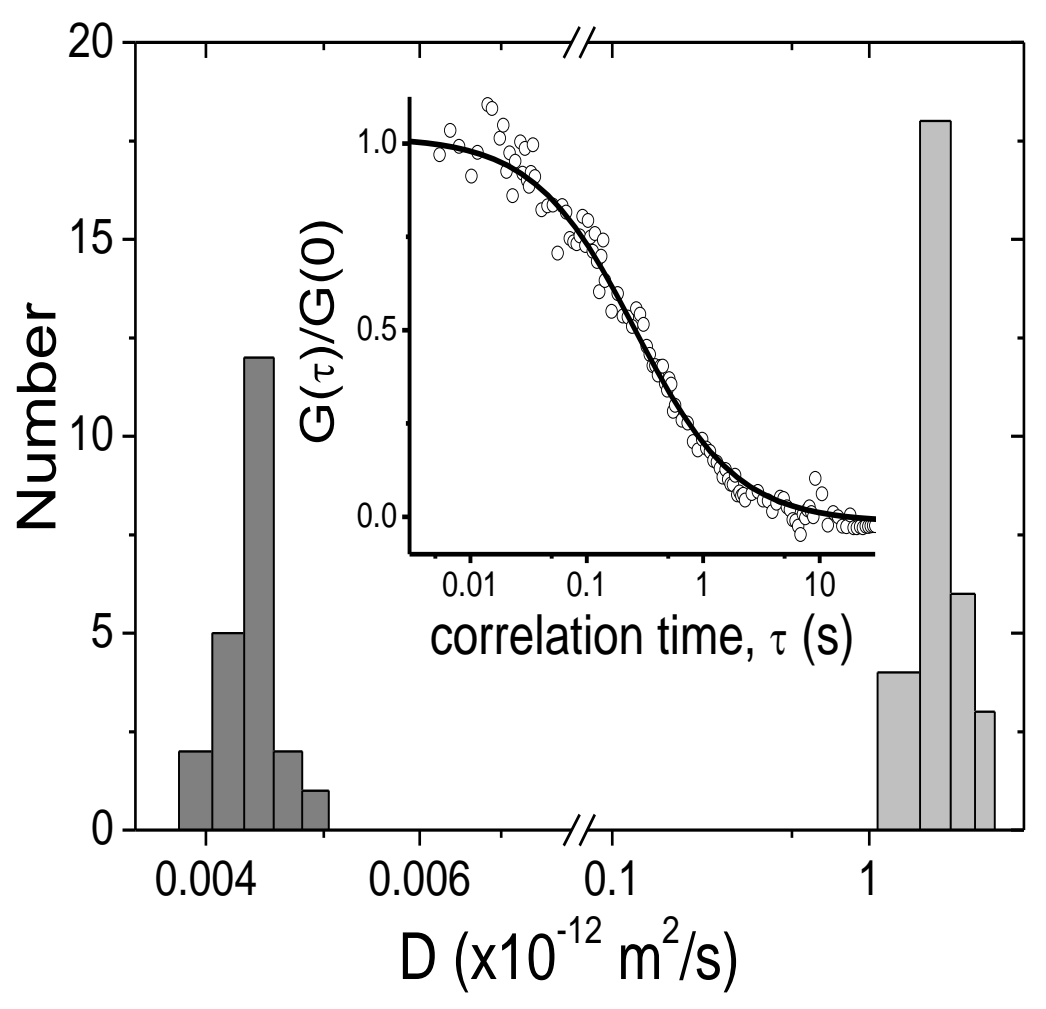


Figure 2

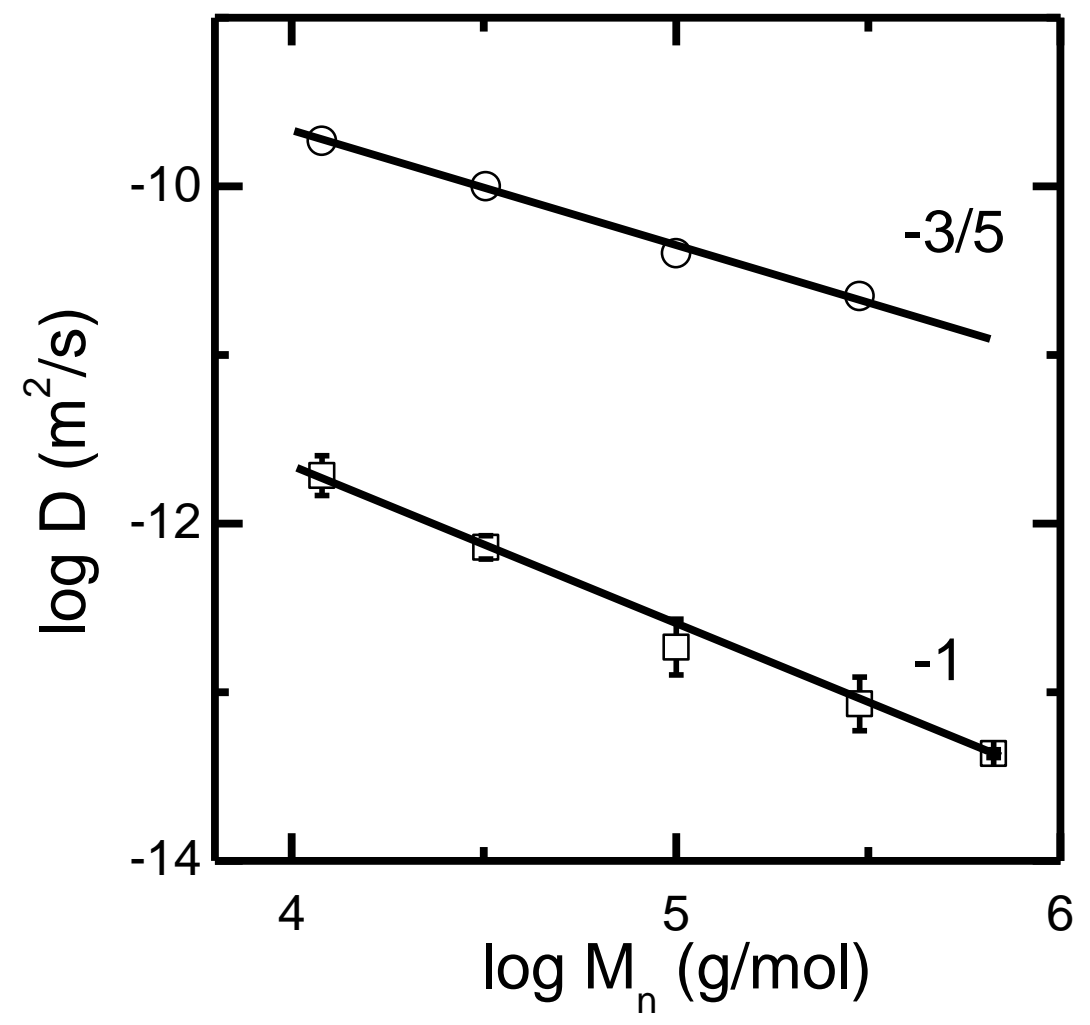


Figure 3

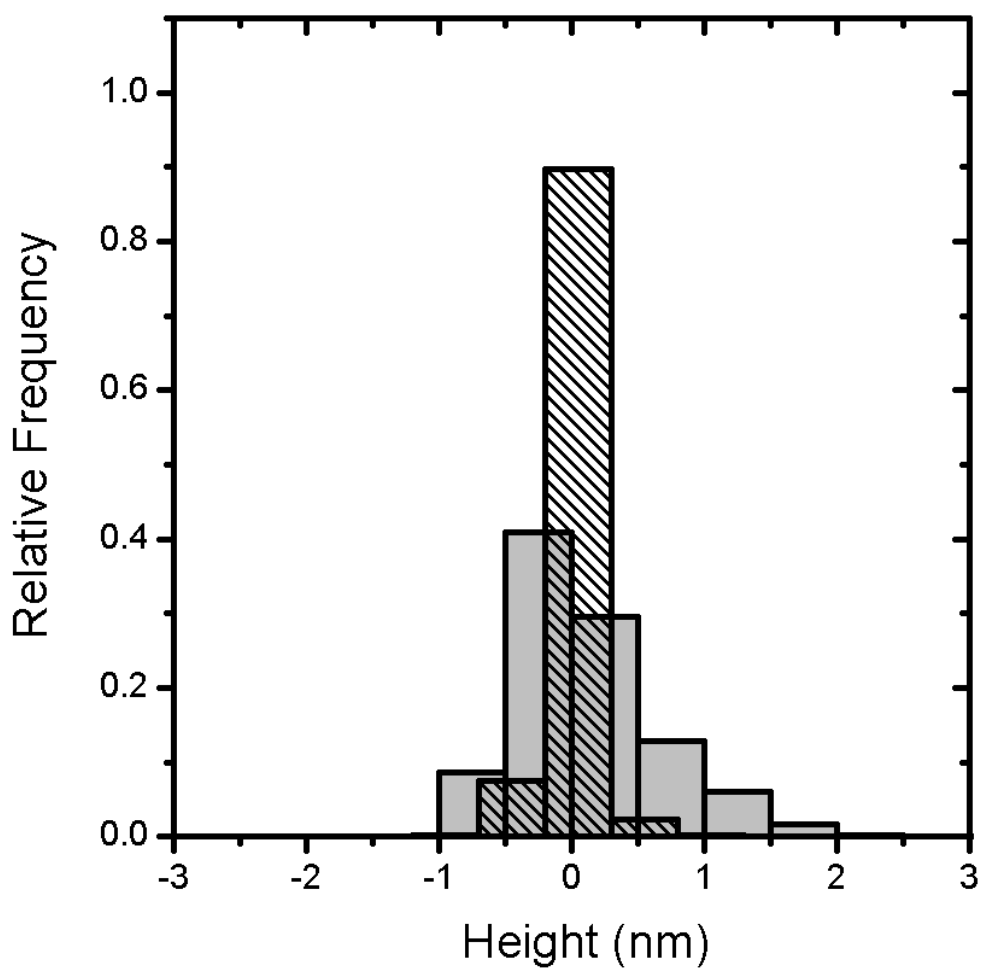


Figure 4

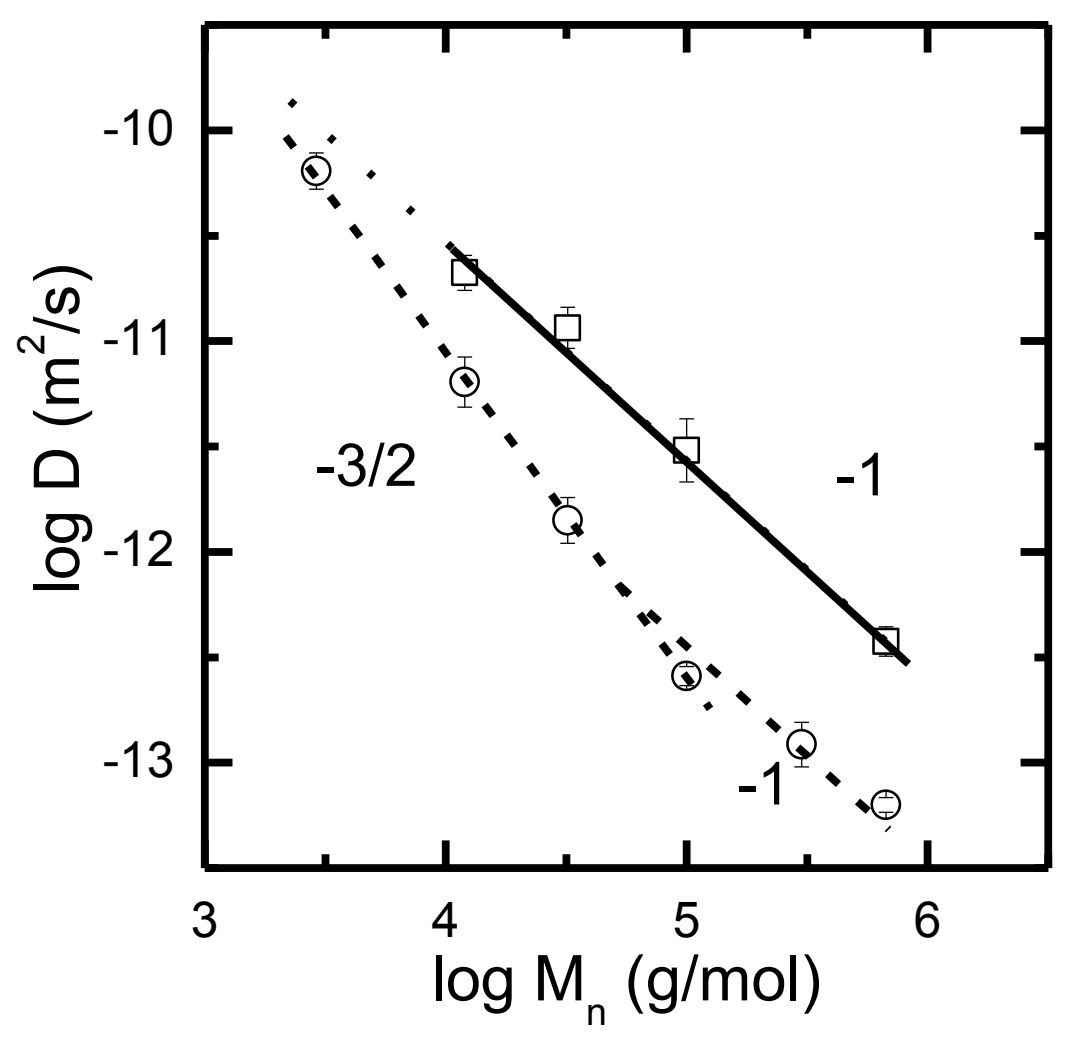

